\title{
The Assessment of Major Histocompatibility Complex (MHC) Class-I Expression in Different Neuromuscular Diseases
}

\author{
Maher Kurdi iD ${ }^{1,2}$ \\ Aysha Alshareef ${ }^{3}$ \\ Ahmed K Bamaga ${ }^{4}$ \\ Zahir T Fadel ${ }^{5}$ \\ Moafaq S Alrawaili ${ }^{3}$ \\ Sahar Hakamy ${ }^{2}$ \\ Fawaz Mohamed (D) 1,2 \\ Ahmad R Abuzinadah (iD ${ }^{3}$ \\ Bassam MJ Addas 5 \\ Nadeem Shafique Butt (iD ${ }^{6}$ \\ 'Department of Pathology, Faculty of \\ Medicine, King Abdulaziz University, \\ Rabigh, Saudi Arabia; ${ }^{2}$ Neuromuscular Unit, \\ Roya Medical Specialized Laboratories, \\ King Abdulaziz University, Jeddah, Saudi \\ Arabia; ${ }^{3}$ Department of Internal Medicine, \\ King Abdulaziz University Hospital and \\ Faculty of Medicine, King Abdulaziz \\ University, Jeddah, Saudi Arabia; \\ ${ }^{4}$ Neurology Division, Department of \\ Pediatrics, Faculty of Medicine, King \\ Abdulaziz University and Hospital, Jeddah, \\ Saudi Arabia; ${ }^{5}$ Department of Surgery, \\ Faculty of Medicine, King Abdulaziz \\ University, Jeddah, Saudi Arabia; \\ ${ }^{6}$ Department of Family Medicine and \\ Community, Faculty of Medicine, King \\ Abdulaziz University, Rabigh, Saudi Arabia
}

Correspondence: Maher Kurdi Department of Pathology, Faculty of Medicine in Rabigh, King Abdulaziz University, Jeddah, Saudi Arabia Email Ahkurdi@kau.edu.sa
Background: Major histocompatibility complex (MHC) class-1 antigen is a glycoprotein expressed in all nucleated cells. The aim of this study was to assess MHC class-I expression in different neuromuscular diseases.

Methods: The authors reviewed the data of 54 patients with neuromuscular diseases. Anti MHC class-I antibody was performed on the frozen muscle tissues using immunohistochemistry. MHC class-I was scored based on its expression on muscle fibers (0: normal, 1: expression $<5$ fibers, 2: expression in 5-10 fibers, 3: expression in $>10$ fibers). The pattern was only assessed in cases with MHC class-I scored 3 as: (1: Sarcocapillary, 2: Sarcocapillary and necrotic fibers, 3: Perifascicular). The relationship between MHC classI expression and neuromuscular diseases was statistically analyzed.

Results: The mean age of the patients was $39.1 \pm 18.5$ years. Around $50 \%$ of patients showed normal CK levels and $5 \%$ of the cases showed elevated CK levels. There was a significance difference in MHC class-I expression between cases with normal and elevated CK levels when MHC class-I score was $3(p=0.020)$. There was a significant difference in MHC class-I expression among different neuromuscular diseases $(p<0.001)$. All cases with idiopathic inflammatory myopathies (IIMs) have expressed MHC class-I in more than 10 fibers. MHC class-I was expressed in 15 cases of non-IIMs.

Conclusion: MHC class-I cannot be solely used as a biomarker to distinguish IIMs from non-IIMs. The presence of MHC class-I molecules in non-IIMs might be related to immunoproteasomes mechanism. Further studies, with different muscle proteins expression and genomic sequencing, must be conducted to understand the role of MHC Class-I in neuromuscular diseases.

Keywords: MHC class-I, neuromuscular diseases, inflammatory myopathy, muscle biopsy

\section{Introduction}

The major histocompatibility complex (MHC) class-I antigen is an integral membrane glycoprotein normally expressed in all nucleated cells. ${ }^{1}$ In human, normal muscle tissue does not express MHC class-I and the expression is usually seen in normal endomysial capillaries. ${ }^{1}$ MHC class-I plays an important role in both initiating and continuing the muscle damage. It is triggered by a variety of factors, only a prolonged upregulation with abnormal expression of sarcolemmal proteins or predisposing genetic background in certain individuals may cause muscle inflammation. ${ }^{1}$ Recent studies suggested that the lack of MHC class-I expression in normal muscle may play an important role in protecting striated muscle from 


\section{Graphical Abstract}

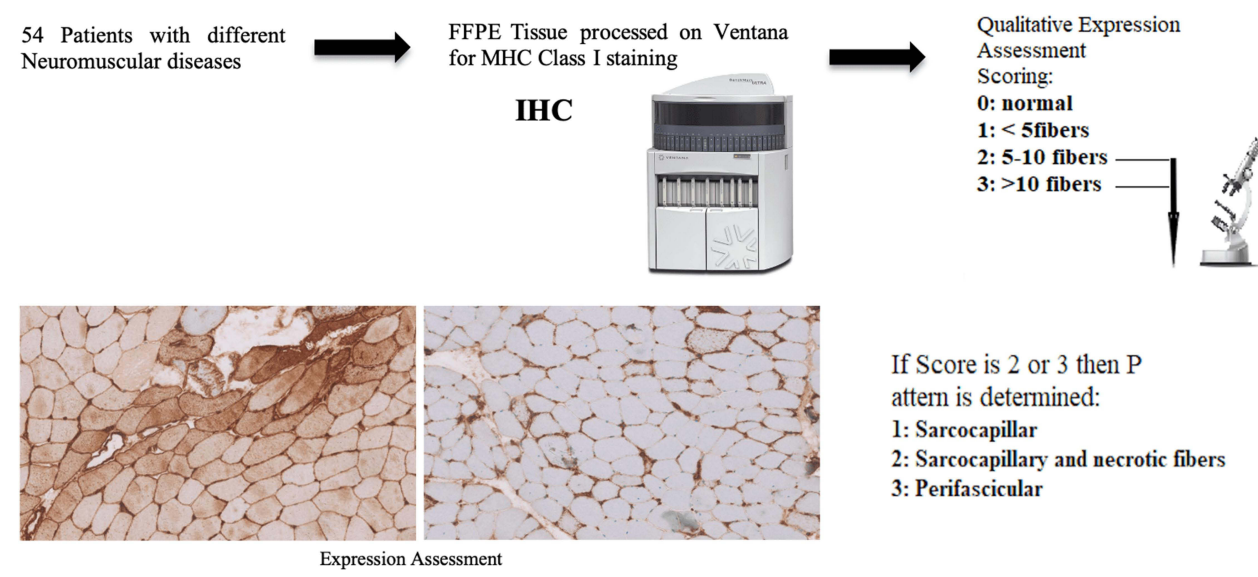

apoptosis. The exact cause of increased MHC class-I expression and its specific role in the pathogenesis of different myopathic diseases, particular myositis, remains unidentified. $^{2}$

Since the last 30 years, MHC class-I expression has been detected in different variants of idiopathic inflammatory myopathies (IIMs). ${ }^{1,3,4}$ Many Pathologists or Neurologists used it as a sensitive marker to distinguish IIMs from non-IIMs. van der Pas et al, evaluated 208 muscle biopsy samples, stained with MHC class-I antigen, and they found that there was no MHC class-I expression in metabolic, congenital, and neurogenic disorders as well as healthy controls. ${ }^{3}$ However, they found that MHC classI was expressed in 60-90\% of muscle biopsies from patients with IIMs. Nagappa et al, also demonstrated that, in patients with IIMs, the MHC class-I was upregulated along the membrane of all muscle fibers. ${ }^{1}$ Some of the inflammatory cells were labeled with MHC class-I in the endomysium and perimysium, whereas sarcoplasmic staining was not identified in any muscle fibers including the regenerating and necrotic fibers. ${ }^{1}$

MHC class-I has also been detected in non-IIMs, such as muscular dystrophies. ${ }^{2,3}$ Although it is not yet fully investigated, this finding may eliminate the theory that MHC class-I is a diagnostic marker for IIMs. van der Pas et al, found that MHC class-I was expressed in $11 \%$ of the biopsies taken from patients with muscular dystrophy. ${ }^{3}$ There were $6 \%$ false negative while false positive results were $15 \%$. The main cause of false negative results was long-term immunosuppressive treatment prior to biopsy. Nagappa et al, also reported that MHC class-I expression may be less sensitive method to differentiate muscular dystrophies with inflammation from IIMs. The expression was mainly found in the sarcolemma in both muscular dystrophies as well as IIMs. ${ }^{1}$ These findings were also not conclusive particularly in IIMs that lack inflammation. van der Pas et al, reported MHC class-I expression in 6 muscle biopsy patients of IIM with absent inflammatory infiltrates. ${ }^{3}$ This explains the fact that MHC class-I is diffusely distributed throughout the biopsy and not affected by potential sampling errors. ${ }^{3}$ Iwasa et al, also reported that some Myasthenia Gravis (MG) patients expressed MHC class-I and MHC class-II in their muscle fibers. ${ }^{5}$ Moreover, Yin et al, found that some patients with dysferlinopathy (Limb girdle muscular dystrophy-type IIB) showed MHC class-I expression in their muscle fibers. ${ }^{6}$

Up-to-date, MHC class-I molecules have never been explored scientifically in different neuromuscular diseases, in addition, it was not clear whether MHC class-I expression can assist in distinguishing IIMs from non-IIMs. This study is aimed to explore the expression of MHC class-I antigen in different neuromuscular diseases including IIMs, and to investigate if MHC class-I can be used as a diagnostic marker for IIMs.

\section{Materials and Methods}

\section{Patients' Stratification}

This study included 54 patients with different neuromuscular diseases, diagnosed by our neuromuscular unit at 
Roya Medical Specialized Laboratories at King Abdulaziz University in the period between 2020 and 2021. The study was ethically approved by the National Biomedical Ethics Committee at King Abdulaziz University (HA-02$\mathrm{J}-008$ ), in which the samples were restricted to Roya Medical Specialized Laboratories. All participants were informed about the purpose of the study, in accordance with the Declaration of Helsinki. Clinical data were retrieved from our medical lab records including patients' age at diagnosis, gender, creatinine kinase (CK) level, electromyographic (EMG) findings, muscle pathology findings, and final neuromuscular diagnoses.

\section{Histopathology}

Hematoxylin and eosin $(\mathrm{H} \& \mathrm{E})$ slide of each patient sample was examined by a certified neuromuscular pathologist (MK) to re-evaluate the histopathological diagnosis.

\section{Immunohistochemistry (IHC) Protocol}

An additional one unstained section $(9-\mu \mathrm{m})$ of frozen muscle tissue was prepared for immunohistochemistry (IHC). The IHC assay, using anti-HLA Class-I monoclonal antibody (clone W6/32-Cat\# Ab22432, Abcam) was performed with the ultraView DAB detection Kit from Ventana on a BenchMark XT automated stainer (Ventana, Tucson, AZ). The entire assay procedure consisted of antibody optimization using a dilution ranging between 1:400 and 1:500 followed by primary tissue incubation for 16 minutes at $37^{\circ} \mathrm{C}$. The slides were counterstained with haematoxylin II for 16 minutes and bluing reagent was used for another 16 minutes. Then, the slides were removed from the slide stainer and immersed into successive alcohol buffers at different concentrations for 3 minutes.

\section{Assessment of MHC Class-I Expression (Scoring and Pattern)}

MHC class-I is normally expressed in all nucleated cells of the endomysial capillaries and muscle fibers without sarcoplasmic expression (Figure 1A). If the expression expands to involve the muscle fiber sarcoplasm or densely expresses the endomysial capillaries, the staining is defined as abnormal. The sarcoplasmic staining of muscle fibers has been scored into three categories: (1) expression in $<5$ muscle fibers, (2) expression in 5-10 muscle fibers, and expression involves more than 10 muscle fibers excluding regenerating fibers. When the expression was scored (2) or (3), the pattern was then assessed. The pattern was categorized as: (1) sarcocapillary staining, (2) sarcocapillary with necrotic fibers, or (3) perifascicular staining (Figure 1B-D). MHC class-I staining showed various expression patterns in different neuromuscular diseases (Figure 2).

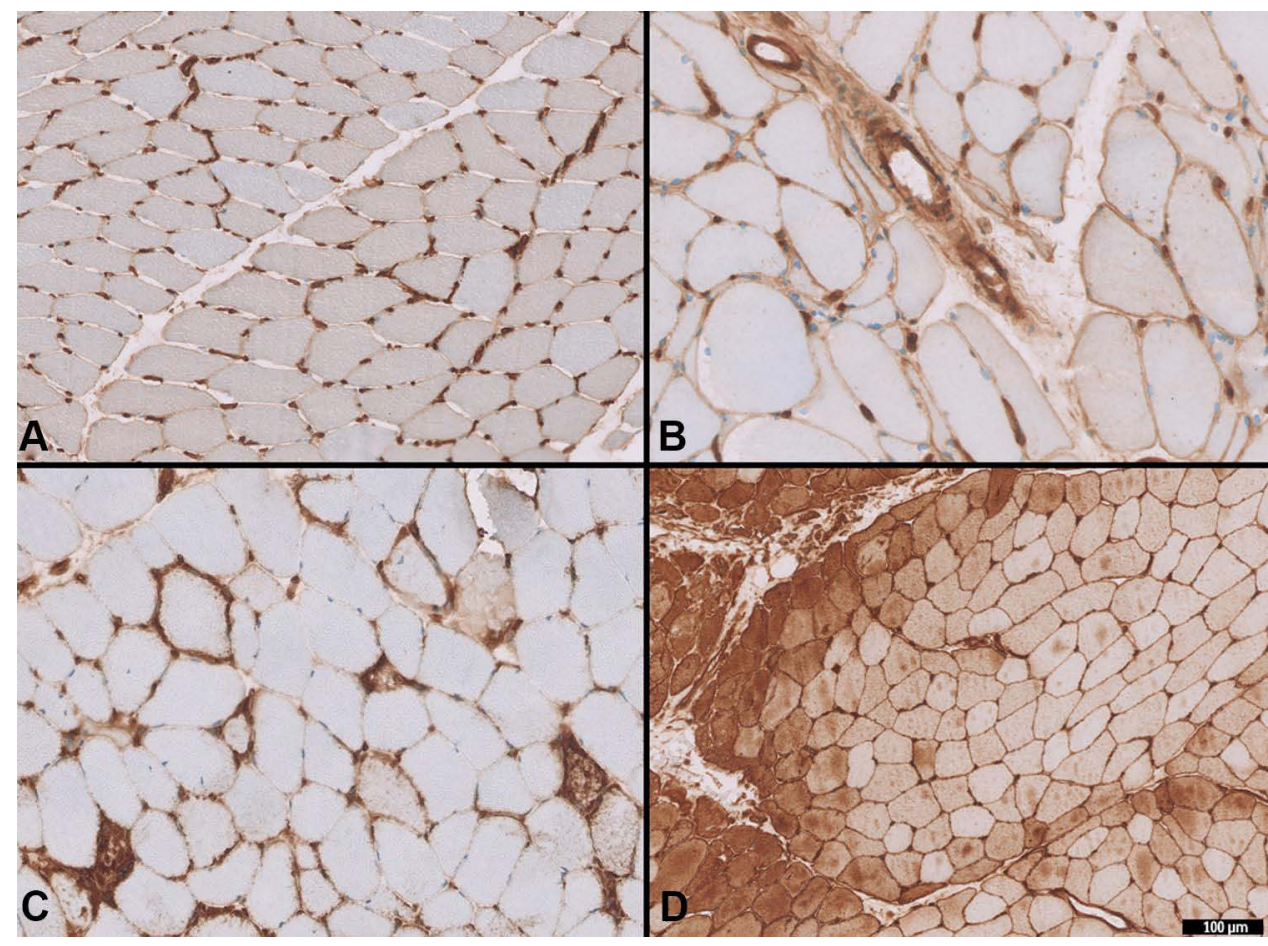

Figure I MHC class-I expression scores. (A) Score 0, normal expression of endomysial capillaries, (B) Score I, sarcocapilary expression, (C) sarcocapilary expression with necrotic fibers expression, (D) perifascicular expression. Magnification $\times 100 \mu \mathrm{m}$. 


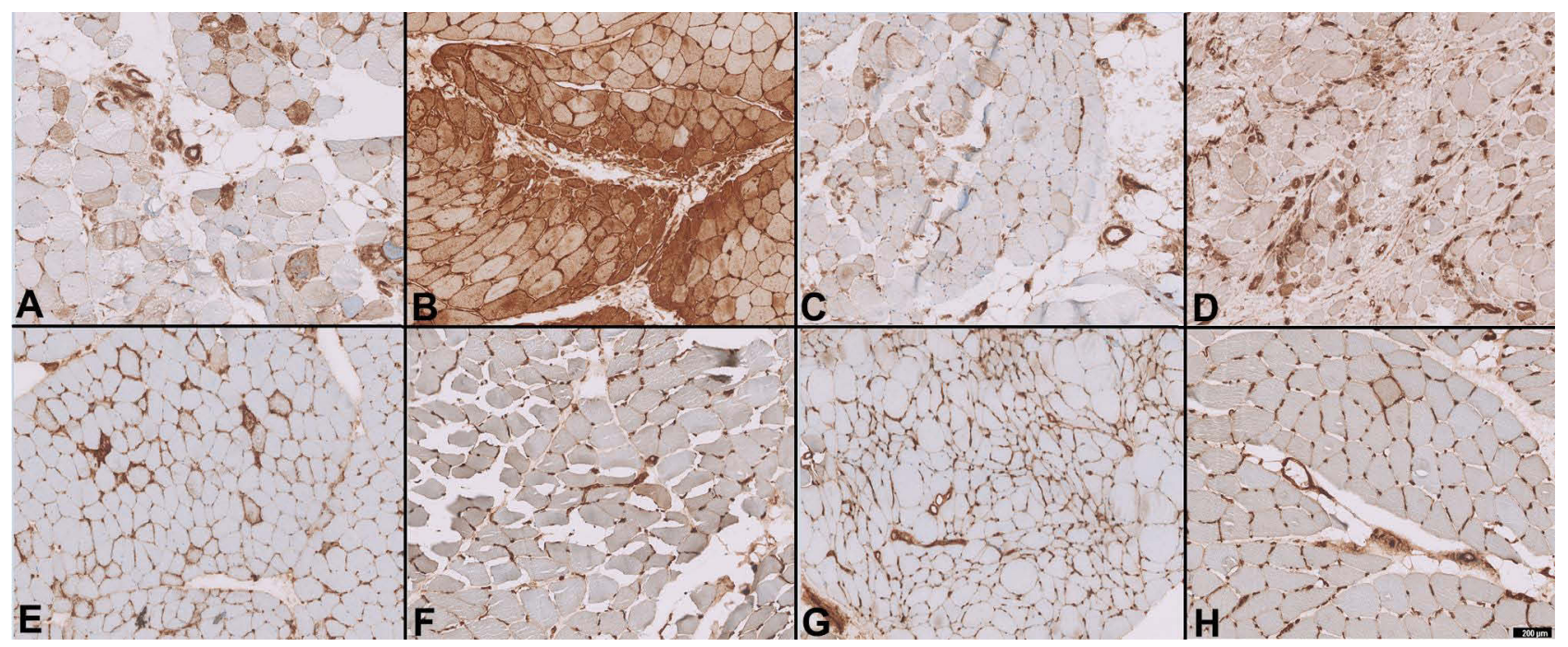

Figure 2 MHC class-I expression in different neuromuscular diseases. (A) Idiopathic inflammatory myopathy (IIM), (B) Dermatomyositis, (C) Muscular dystrophy, (D) Metabolic Myopathy (POMPE), (E) Mitochondrial myopathy, (F) Myasthenia Gravis Disease, (G) Chronic denervation atrophy, (H) None-specific fibers atrophy. Magnification $\times 200 \mu \mathrm{m}$.

\section{Statistical Methods}

The data were described as frequencies and percentages. The Fisher's exact test was used to test the relationship between CK-level, MHC class-I expression score and pattern, and histopathological diagnoses. We also used Z-test to calculate the proportion between different variables. The overall P-value was used to test the significance. P-value less than 0.05 or 0.001 was considered significant. All statistical analyses were performed using the IBM SPSS1 ver. 24 statistical software programs (SPSS Inc., Chicago, IL).

\section{Results}

This study included 54 patients with different neuromuscular diseases or conditions. The mean age of the patients was 39.1 \pm 18.5 years. There were more females $(\mathrm{n}=30)$ than males $(\mathrm{n}=24)$. Around $50 \%$ of patients showed normal CK levels (less $300 \mathrm{IU} / \mathrm{L}$ ) and $5 \%$ of the cases showed elevated CK levels $(>300 I U / L)$. The EMG of the patients showed myopathic features $(53.7 \%, \mathrm{n}=29)$ neuropathic or neuromyopathic change $(11.1 \%, \mathrm{n}=6)$ each, and irritative myopathic change $(16.7 \%, n=9)$. Around $7 \%(n=4)$ of the cases showed nonspecific findings. Muscle biopsy of the patients showed myopathic change $(38.8 \%, \mathrm{n}=21)$, neuropathic change $(16.7 \%$, $\mathrm{n}=9)$, neuromyopathic change $(9.3 \%, \mathrm{n}=5)$, inflammatory myopathic change $(13 \%, n=7)$, and other non-specific change $(22.2 \%, \mathrm{n}=12)$. Necrotic fibers were found in $5.6 \%$ and regenerated fibers were found in $9.3 \%$ however, $42.6 \%$ cases showed both necrotic and regenerated fibers. Patients have been finally diagnosed with specific neuromuscular diseases (inflammatory and non-inflammatory myopathies), which included: axial myopathy $(\mathrm{n}=1)$, chronic denervation atrophy $(\mathrm{n}=9)$, hereditary myopathies $(\mathrm{n}=6)$, inflammatory myopathies $(\mathrm{n}=12)$, metabolic myopathy $(\mathrm{n}=3)$, mitochondrial myopathies $(\mathrm{n}=1)$, Myasthenia Gravis disease $(\mathrm{n}=1)$, muscular dystrophy $(n=7)$, non-inflammatory necrotizing myopathy $(n=4)$, rare atrophic fibers $(\mathrm{n}=10)$. For inflammatory myopathies, 3 cases were adult-type dermatomyositis, 1 case inclusion body myositis, and the rest of cases were nonspecific myositis. Hereditary myopathies included 3 cases myofibrillar myopathies (Filamin $C$ mutation), Titinopathy, and two cases of Klippel-Feil deformity with myopathy (MYO18B defect). These cases were diagnosed through Whole-Exom Sequencing (WESeq). Tables 1-3 describe the demographic and statistical distribution of the data.

\section{Statistical Analysis}

The Relationship Between CK Levels and MHC Class-I Scores

MHC class-I was normally expressed in the endomysial capillaries of $63 \%(n=17)$ of the patients with normal CK level and $33.3 \%(n=9)$ of patients with elevated CK level (Table 2). MHC class-I did not show a significant difference in the expression of muscle fibers (5-10 fibers) between cases with normal or elevated CK levels. However, the significance difference in expression was obviously observed between cases with normal and 
Table I Demographic Distribution of the Data in This Study

\begin{tabular}{|l|l|}
\hline & Overall (n=54) \\
\hline Age & \\
Mean (SD) & $39.1(18.5)$ \\
Range & $4.0-77.0$ \\
Gender & \\
Female & $30(55.6 \%)$ \\
Male & $24(44.4 \%)$ \\
CK Level & \\
Normal & $27(50.0 \%)$ \\
Elevated & $27(50.0 \%)$ \\
EMG Finding & \\
Myopathic & $29(53.7 \%)$ \\
Neuropathic & $6(11.1 \%)$ \\
Neuromyopathic & $6(11.1 \%)$ \\
Irritative myopathic & $9(16.7 \%)$ \\
Non-Specific & $4(7.4 \%)$ \\
Main Muscle Pathological Finding & \\
Myopathic change & $21(38.8 \%)$ \\
Neuropathic change & $9(16.7 \%)$ \\
Neuromyopathic change & $5(9.3 \%)$ \\
Inflammatory myopathy & $7(13.0 \%)$ \\
Nonspecific change & $12(22.2 \%)$ \\
Muscle Degeneration & \\
Necrotic fibers & $3(5.6 \%)$ \\
Regenerated fibers & $5(9.3 \%)$ \\
Necrotic and regenerated fibers & $23(42.6 \%)$ \\
None & $23(42.6 \%)$ \\
\hline
\end{tabular}

elevated CK levels when MHC class-I score was 3 (>10 fibers staining) (overall $p=0.020$ ) (Table 2). The cases, in which their MHC class-I was scored-3, was not statistically differed in expression pattern between necrotic and regenerated muscle fibers $(p=0.752)$.

MHC Class-I Expression Scores and Patterns Among Neuromuscular Diseases

There was a statistically significant difference in MHC class-

I expression among different types of neuromuscular diseases (overall $\mathrm{p}<0.001$ ). MHC class-I expression was normally expressed in the endomysial capillaries of chronic denervation atrophy $(\mathrm{n}=6)$, metabolic myopathy $(\mathrm{n}=1)$, hereditary myopathy $(\mathrm{n}=6)$, myopathic dystrophy $(\mathrm{n}=4)$, and cases with non-specific fibers atrophy $(\mathrm{n}=8)$. All cases $(12 /$ 12) of inflammatory myopathies (IIMs) expressed MHC class-I in more than 10 fibers (score 3) (Table 3). Cases such as myopathic dystrophy $(\mathrm{n}=2)$ and metabolic myopathy $(n=2)$, where focal inflammatory cells were seen in their muscle fibers, have showed a score 2 or 3 of MHC class-I expression (Table 3). MHC class-I expression of sarcocapillaries and necrotic fibers were mainly found in IIMs as well as 4 cases of non-IIMs including mitochondrial, metabolic, and non-inflammatory necrotizing myopathies (Table 4). Cases with perifascicular MHC-class I expression were predominantly associated with dermatomyositis.

\section{Discussion}

The role of major histocompatibility complex (MHC) molecules in human immunity has been studied since their discovery in $1936 .^{7}$ MHC class-I molecules are loaded with endogenous peptides, generated by the proteasome, and imported into the endoplasmic reticulum by the heterodimeric transporter. It was proposed to be controlled via the NF- $\mathrm{KB}$ binding Enhancer A region and interferon (IFN)-sensitive response element (ISRE) motif in the promoter regions. ${ }^{8}$ These regions certainly contribute to MHC class-I expression. MHC class-I molecules are polymorphic in human cells, which cause a large diversity of peptides and untranslated regions to be presented by MHC class-I and associated with different expression levels. ${ }^{9}$ These two variables influence the immune responses among different patients which clarify why MHC class-I molecules are often associated with diseases like infections, autoimmunity, and cancer. Bhattarai et al, suggested that immunoproteasome is involved in pathologic MHC class-I expression and in the maintenance of myokine production in

Table 2 The Relationship Between MHC Class-I Expression in Muscle Fibers and CK Level. There Was a Significant Difference in Expression Between Cases with Normal and Elevated CK Level (Overall p-value <0.020)

\begin{tabular}{|c|c|c|c|c|}
\hline \multirow{2}{*}{} & \multicolumn{2}{|c|}{ CK Level } & & \\
\cline { 2 - 5 } & Normal (n=27) & Elevated (n=27) & Total (n=54) & *P-value \\
\hline MHC Class I Expression Score & & & & $0.020^{\mathrm{a}}$ \\
0 & $17.0(63.0 \%)$ & $9.0(33.3 \%)$ & $26.0(48.1 \%)$ & $0.029^{\mathrm{b}}$ \\
1 & $3.0(11.1 \%)$ & $1.0(3.7 \%)$ & $4.0(7.4 \%)$ & $0.299^{\mathrm{b}}$ \\
2 & $1.0(3.7 \%)$ & $1.0(3.7 \%)$ & $2.0(3.7 \%)$ & $1.000^{\mathrm{b}}$ \\
3 & $6.0(22.2 \%)$ & $16.0(59.3 \%)$ & $22.0(40.7 \%)$ & $0.006^{\mathrm{b}}$ \\
\hline
\end{tabular}

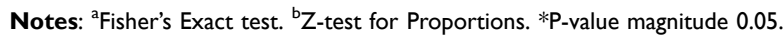


Table 3 MHC Class-I Expression in Different Types of Neuromuscular Diseases. Overall, There Was a Significant Difference in MHC Also I Expression Scores Among All Neuromuscular Diseases

\begin{tabular}{|c|c|c|c|c|c|c|}
\hline & \multicolumn{4}{|c|}{ MHC Class I Expression Score } & \multirow[b]{2}{*}{ Total $(n=54)$} & \multirow[b]{2}{*}{ *P-value } \\
\hline & $0(n=26)$ & I $(n=4)$ & $2(n=2)$ & $3(n=22)$ & & \\
\hline Neuromuscular Diagnosis & & & & & & $<0.001^{\mathrm{a}}$ \\
\hline Axial Myopathy & $1.0(3.8 \%)$ & $0.0(0.0 \%)$ & $0.0(0.0 \%)$ & $0.0(0.0 \%)$ & $1.0(1.9 \%)$ & c \\
\hline Chronic denervation atrophy & $6.0(23.1 \%)$ & $1.0(25.0 \%)$ & $0.0(0.0 \%)$ & $2.0(9.1 \%)$ & $9.0(16.7 \%)$ & $0.585^{\mathrm{b}}$ \\
\hline Hereditary Myopathy & $6.0(23.1 \%)$ & $0.0(0.0 \%)$ & $0.0(0.0 \%)$ & $0.0(0.0 \%)$ & $6.0(11.1 \%)$ & c \\
\hline Inflammatory Myopathy & $0.0(0.0 \%)$ & $0.0(0.0 \%)$ & $0.0(0.0 \%)$ & $12.0(54.5 \%)$ & $12.0(22.2 \%)$ & c \\
\hline Metabolic Myopathy & $\mathrm{I} .0(3.8 \%)$ & $0.0(0.0 \%)$ & $1.0(50.0 \%)$ & $1.0(4.5 \%)$ & $3.0(5.6 \%)$ & $0.044^{b}$ \\
\hline Mitochondrial myopathy & $0.0(0.0 \%)$ & $0.0(0.0 \%)$ & $0.0(0.0 \%)$ & I.0 (4.5\%) & $1.0(1.9 \%)$ & $0.078^{\mathrm{b}}$ \\
\hline Myasthenia Gravis disease & $0.0(0.0 \%)$ & $1.0(25.0 \%)$ & $0.0(0.0 \%)$ & $0.0(0.0 \%)$ & $1.0(1.9 \%)$ & c \\
\hline Myopathic Dystrophy & $4.0(15.4 \%)$ & $1.0(25.0 \%)$ & $0.0(0.0 \%)$ & $2.0(9.1 \%)$ & $7.0(13.0 \%)$ & c \\
\hline NINM & $0.0(0.0 \%)$ & $0.0(0.0 \%)$ & $1.0(50.0 \%)$ & $3.0(13.6 \%)$ & $4.0(7.4 \%)$ & $0.186^{b}$ \\
\hline Rare atrophic fibers & $8.0(30.8 \%)$ & $1.0(25.0 \%)$ & $0.0(0.0 \%)$ & $1.0(4.5 \%)$ & $10.0(18.5 \%)$ & $0.06 \mathrm{I}^{\mathrm{b}}$ \\
\hline
\end{tabular}

Notes: ${ }^{a}$ Fisher's Exact test. ${ }^{\mathrm{b}}$ Z-test for Proportions. ${ }^{\mathrm{C}}$ This category is not used in comparisons because its proportion is $0 .{ }^{*} \mathrm{P}$-value magnitude $0.00 \mathrm{I}$. Abbreviation: NINM, non-inflammatory necrotizing myopathy.

Table 4 The Pattern of MHC Class-I Expression in Different Types of Neuromuscular Diseases When the MHC Class-I Expression Score 2 and Above

\begin{tabular}{|c|c|c|c|c|}
\hline & \multicolumn{2}{|c|}{ MHC Class I Expression Pattern } & \multirow[b]{2}{*}{ Total } & \multirow{3}{*}{ *P-value } \\
\hline & $\mathbf{I}$ & $\geq 2$ & & \\
\hline & $(n=13)$ & $(n=I I)$ & $(n=24)$ & \\
\hline Histopathological Diagnosis & & & & $0.540^{\mathrm{a}}$ \\
\hline Chronic denervation atrophy & $2.0(15.4 \%)$ & $0.0(0.0 \%)$ & $2.0(8.3 \%)$ & c \\
\hline Inflammatory Myopathy & $5.0(38.5 \%)$ & $7.0(63.6 \%)$ & $12.0(50 \%)$ & $0.219^{b}$ \\
\hline Metabolic Myopathy & $1.0(7.7 \%)$ & $1.0(9.1 \%)$ & $2.0(8.3 \%)$ & $0.902^{\mathrm{b}}$ \\
\hline Mitochondrial myopathy & $0.0(0.0 \%)$ & $1.0(9.1 \%)$ & $1.0(4.2 \%)$ & c \\
\hline Myopathic Dystrophy & $2.0(15.4 \%)$ & $0.0(0.0 \%)$ & $2.0(8.3 \%)$ & c \\
\hline NINM & $2.0(15.4 \%)$ & $2.0(18.2 \%)$ & $4.0(16.7 \%)$ & $0.855^{\mathrm{b}}$ \\
\hline Rare atrophic fibers & $1.0(7.7 \%)$ & $0.0(0.0 \%)$ & $\mathrm{I} .0(4.2 \%)$ & c \\
\hline
\end{tabular}

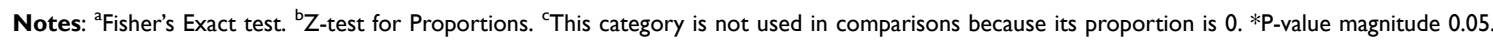
Abbreviation: NINM, non-inflammatory necrotizing myopathy.

IIMs. Thus, induction of the immunoproteasome was identified as a mechanism of inflammation in IIMs. ${ }^{10}$ Our study found that MHC class-I was diffusely expressed in the muscle fibers of the whole IIMs (Table 3). Rodriguez Cruz et al, found that MHC class-I expression had a high sensitivity in biopsies from patients with inflammatory myopathy but a very low specificity, as it was also common in other non-IIMs and neurogenic atrophies. ${ }^{12}$

The expression of MHC class-I in myopathic dystrophy groups is rarely documented in the literature. McDouall et al, investigated MHC class-I and II in 200 skeletal muscle biopsies. They found that MHC was not expressed in normal muscle but strongly expressed in IIMs and Duchene muscular dystrophy (DMD). ${ }^{13}$ Sundaram et al, studied various types of dystrophies including DMD, Becker muscular dystrophy
(BMD), LGMD, and myotonic dystrophy (MD), and they found uniform absence of MHC class-I expression in muscle fibers including the fibers adjacent to inflammatory cell infiltrates whereas the expression was only found in the regenerating fibers. ${ }^{4}$ Yin et al, demonstrated the involvement of immune factors (MHC) in the pathogenesis of dysferlinopathy. ${ }^{6}$ They also found that MHC class-I was expressed in 10/14 cases of LGMD type IIB and 2 cases of DMD. It is possible that dysferlin interacts with significant immunological pathways. ${ }^{11}$ Our study demonstrated that MHC class-I was expressed in three cases of myopathic dystrophy; two of them were associated with widespread staining involving mainly sarcocapillaries excluding necrotic fibers. These two cases had focal areas of inflammation mainly macrophages. None of hereditary myopathic patients (myofibrillar myopathy or 
Klippel Feil myopathies) in our study were found to have MHC class-I expression. For MGD, we had one case with minimal expression (score 1). It was not clear why MGD muscle fibers expresses MHC class-I or II. This finding was typical to Iwasa et al finding. ${ }^{5}$ Cases where marked neuropathic changes are seen in muscle biopsies (such as chronic denervation atrophy) have demonstrated MHC class-I expression in sarcocapillaries. This is likely attributed to the presence of severely atrophic and regenerated muscle fibers. Two of these cases were proven to be amyotrophic lateral sclerosis (ALS).

The mechanism of MHC class-I molecules in various neuromuscular diseases is not well-understood. It may be related to the pathological mechanism of immunoproteasome. The presence of MHC class-I expression in IIMs is likely related to the immunological factors precipitated by different antigens in human bodies. However, other neuromuscular disease entities such muscular dystrophies, mitochondria myopathies, chronic denervation atrophies, MGD, or metabolic myopathies were shown to express MHC class-I. The diversity of peptides and untranslated regions might be associated with these diseases' mechanisms. This absolutely clarifies that MHC class-I can support the diagnosis of IIMs but cannot be solely used to distinguish IIMs from non-IIMs.

\section{Conclusion}

Although MHC class-I was expressed in most of IIMs, it cannot be solely used as a biomarker to distinguish IIMs from non-IIMs. The presence of MHC class-I molecules in non-IIMs might be related to immunoproteasomes mechanism. Further studies, with different muscle proteins expression and genomic sequencing, must be conducted to understand the role of MHC Class-I in neuromuscular diseases.

\section{Data Sharing Statement}

The data that support the findings of this study are available from the corresponding author (MK) upon request.

\section{Ethics Approval and Consent to Participate}

This study was approved by the National Biomedical Ethics Committee of King Abdulaziz University (HA-02J-008) which complies with the guidelines of the "System of ethics of research" prepared by the King Abdulaziz City for Science and Technology and approved by Royal Decree No. M/59 on 24 August 2010.

\section{Consent for Publication}

Obtained. All participants were informed about the purpose of the study, in accordance with the Declaration of Helsinki.

\section{Author Contributions}

All authors made substantial contributions to conception and design, acquisition of data, or analysis and interpretation of data; took part in drafting the article or revising it critically for important intellectual content; agreed to submit to the current journal; gave final approval of the version to be published; and agree to be accountable for all aspects of the work.

\section{Funding}

No funding received for the current study.

\section{Disclosure}

All authors declare no conflicts of interest.

\section{References}

1. Nagappa M, Nalini A, Narayanappa G. Major histocompatibility complex and inflammatory cell subtype expression in inflammatory myopathies and muscular dystrophies. Neurol India. 2013;61 (6):614-621. doi:10.4103/0028-3886.125264

2. Dai TJ, Li W, Zhao QW, Zhao YY, Liu SP, Yan CZ. CD8/MHC-I complex is specific but not sensitive for the diagnosis of polymyositis. J Int Med Res. 2010;38(3):1049-1059. doi:10.1177/ 147323001 003800332

3. van der Pas J, Hengstman GJ, Ter Laak HJ, Borm GF, van Engelen BG. Diagnostic value of MHC class I staining in idiopathic inflammatory myopathies. J Neurol Neurosurg Psychiatry. 2004;75 (1):136-139. PMID: 14707323.

4. Sundaram C, Uppin MS, Meena AK. Major histocompatibility complex class I expression can be used as a diagnostic tool to differentiate idiopathic inflammatory myopathies from dystrophies. Neurol India. 2008;56(3):363-367. doi:10.4103/0028-3886.43457

5. Iwasa K, Kato-Motozaki Y, Furukawa Y, et al. Up-regulation of MHC class I and class II in the skeletal muscles of myasthenia gravis. J Neuroimmunol. 2010;225(1-2):171-174. doi:10.1016/j. jneuroim.2010.04.017

6. Yin X, Wang Q, Chen T, et al. CD4+ cells, macrophages, MHC-I and C5b-9 involve the pathogenesis of dysferlinopathy. Int J Clin Exp Pathol. 2015;8(3):3069-3075. PMID: 26045819.

7. Klein J. Seeds of time: fifty years ago, Peter A: gorer discovered the H-2 complex. Immunogenetics. 1986;24(6):331-338. doi:10.1007/ BF00377947

8. Ting JP, Baldwin AS. Regulation of MHC gene expression. Curr Opin Immunol. 1993;5(1):8-16. doi:10.1016/0952-7915(93)90074-3

9. Parham P, Lomen CE, Lawlor DA, et al. Nature of polymorphism in HLA-A:-B, and-C molecules. Proc Natl Acad Sci U S A. 1988;85 (11):4005-4009. doi:10.1073/pnas.85.11.4005

10. Bhattarai S, Ghannam K, Krause S, et al. The immunoproteasomes are key to regulate myokines and MHC class I expression in idiopathic inflammatory myopathies. $J$ Autoimmun. 2016;75:118-129. doi:10.1016/j.jaut.2016.08.004 
11. Glover L, Brown RH Jr. Dysferlin in membrane trafficking and patch repair. Traffic. 2007;8:785-794. doi:10.1111/j.1600-0854.20 07.00573.x

12. Rodríguez Cruz PM, Luo YB, Miller J, Junckerstorff RC, Mastaglia FL, Fabian V. An analysis of the sensitivity and specificity of MHC-I and MHC-II immunohistochemical staining in muscle biopsies for the diagnosis of inflammatory myopathies. Neuromuscul Disord. 2014;24(12):1025-1035. doi:10.1016/j. nmd.2014.06.436
13. McDouall RM, Dunn MJ, Dubowitz V. Expression of class I and class II MHC antigens in neuromuscular diseases. J Neurol Sci. 1989;89(2-3):213-226. doi:10.1016/0022-510X(89)90023-3

\section{Publish your work in this journal}

Degenerative Neurological and Neuromuscular Disease is an international, peer-reviewed, open access journal focusing on research into degenerative neurological and neuromuscular disease, identification of therapeutic targets and the optimal use of preventative and integrated treatment interventions to achieve improved outcomes, enhanced survival and quality of life for the patient. The manuscript management system is completely online and includes a very quick and fair peer-review system. Visit http://www.dovepress.com/ testimonials.php to read real quotes from published authors. 